\title{
1 \\ Need and demand for treatment
}

\author{
J. C. Davenport, ' R. M. Basker, ${ }^{2}$ J. R. Heath, ${ }^{3}$ J. P. Ralph, ${ }^{4}$ and P-O. Glantz, ${ }^{5}$
}

\author{
This article considers first, factors influencing the \\ need and demand for removable partial dentures \\ (RPDs) and second, the particular requirements \\ and problems of elderly patients related to such \\ treatment.
}

In this part, we will discuss
- Factors influencing the provision of RPDs
- The effect of age and extent of tooth loss
- The shortened dental arch
- Toothwear and root surface caries
- Possible treatment options for the elderly

latter may find that the effort needed to seek out that treatment is just too great.

Recognizing the difference between need and demand prompts the question, 'How many teeth must be lost before a patient seeks prosthetic replacement?' Experience suggests that the answer can vary greatly. On the one hand, the loss of one anterior tooth is usually a powerful motivator for the patient, whereas another patient may have had many posterior teeth extracted before they seek the advice and help of a dentist.

Although the restoration of appearance can be a powerful motivating factor, not every patient will seek treatment following the loss of an anterior tooth. A study of elderly men living in an area where dental treatment was readily available and affordable showed that one in five had at least one unrestored space towards the front of the mouth. In another study, dentists rated the dental appearance of a group of elderly people as less attractive than did the subjects themselves. The dentist must therefore avoid preconceptions and consider the thoughts and wishes of the individual patient before recommending the provision of a denture. The time, effort and understanding taken to make this judgement are likely to prevent unnecessary treatment.

There can thus be a large difference between the perception of need and demand for a prosthesis as an aid to chewing efficiency. Nutritional status is affected by psychological, sociological and economic factors as well as by the effectiveness of the dentition.

\footnotetext{
${ }^{1^{*}}$ Emeritus Professor, University of Birmingham, UK ; ${ }^{2}$ Professor of Dental Prosthetics, University of Leeds and Consultant in Restorative Dentistry, Leeds Teaching Hospitals NHS Trust, Leeds, UK; ${ }^{3}$ Honorary Research Fellow, University of Manchester (Formerly Senior Lecturer in Restorative Dentistry, University of Manchester) and Consultant in Restorative Dentistry, Central Manchester Healthcare Trust, Manchester, UK; ${ }^{4}$ Consultant in Restorative Dentistry, Leeds Teaching Hospitals NHS Trust and Senior Clinical Lecturer, University of Leeds and Honorary Visiting Professor, Centre for Dental Services Studies, University of York, York, UK; ${ }^{5}$ Professor of Prosthetic Dentistry, Consultant in Prosthetic Dentistry, Faculty of Odontology, University of Malmo, Sweden

${ }^{*}$ Correspondence to: 5 Victoria Road, Harborne, Birmingham B17 OAG email:john.davenport@btclick.com

REFEREED PAPER

(C) British Dental Journal 2000; 189: 364-368
}

\section{New publications:}

All the parts which comprise this series (which will be published in the BDJ) have been included (together with a number of unpublished parts) in the book A Cinical Guide to Removable Partial Dentures (ISBN 0-904588-599) and A Clinical Guide to Removable Partial Denture Design (ISBN 0-904588-637). Available from Macmillan on 01256302699

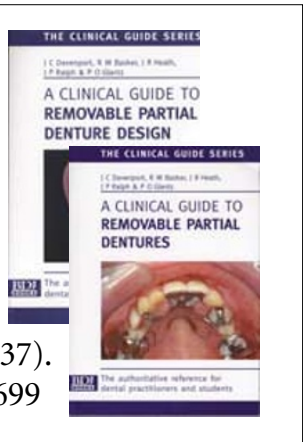


Fig. I

Whether or not a removable partial denture (RPD) is worn by the patient is dependent upon self-motivation. In a UK survey people were asked, 'If you had several missing teeth at the back would you prefer to have an RPD or manage without?' The chart shows that most people would prefer to manage without and that the preference grows stronger with increasing age. It is almost as if the longer the person has managed to avoid dentures the stronger is the wish to do without them.

\section{Fig. 2}

In the same study people were asked, 'Do you find the thought of having a partial denture to replace some of your teeth very upsetting, a little upsetting or not at all upsetting? Overall, only $40 \%$ found the idea of having dentures not at all upsetting. A breakdown of the answers by age is shown in the chart. Again, there is a suggestion that older people tend to find the thought of an RPD more upsetting than do the young.
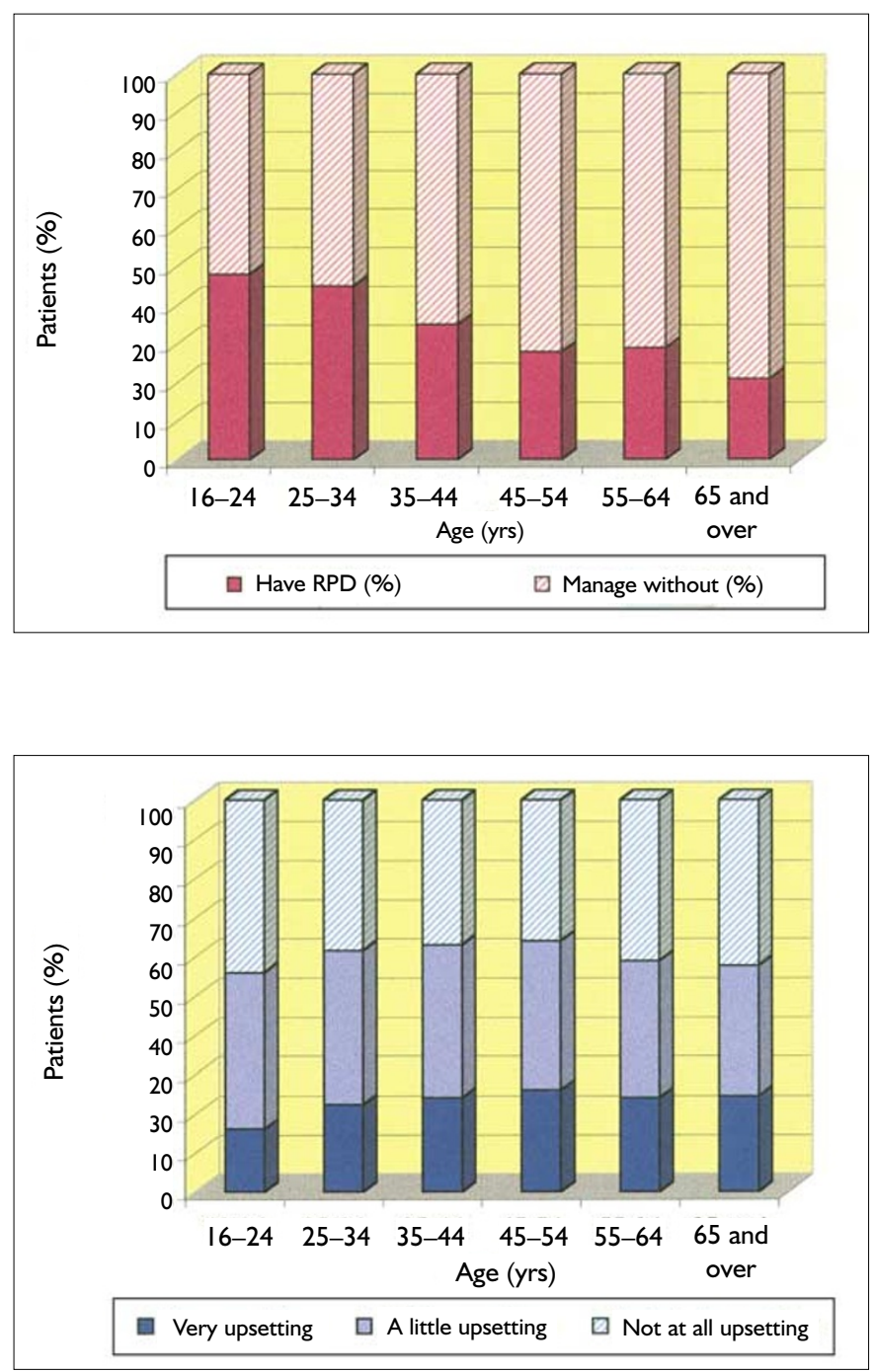

normal function can be satisfied by the presence of natural teeth no further distally than the second premolars.

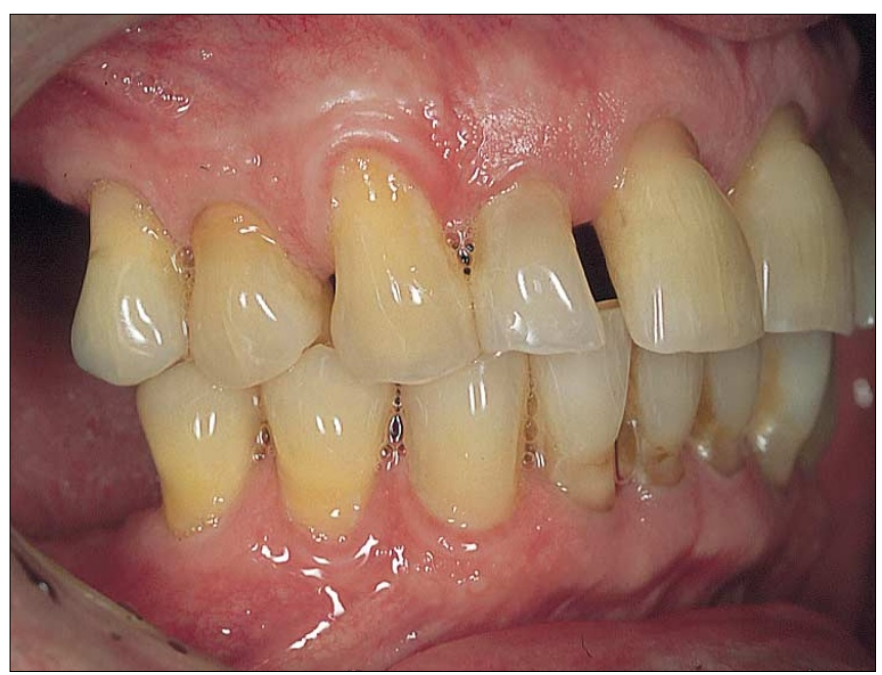




\section{prosthetics}

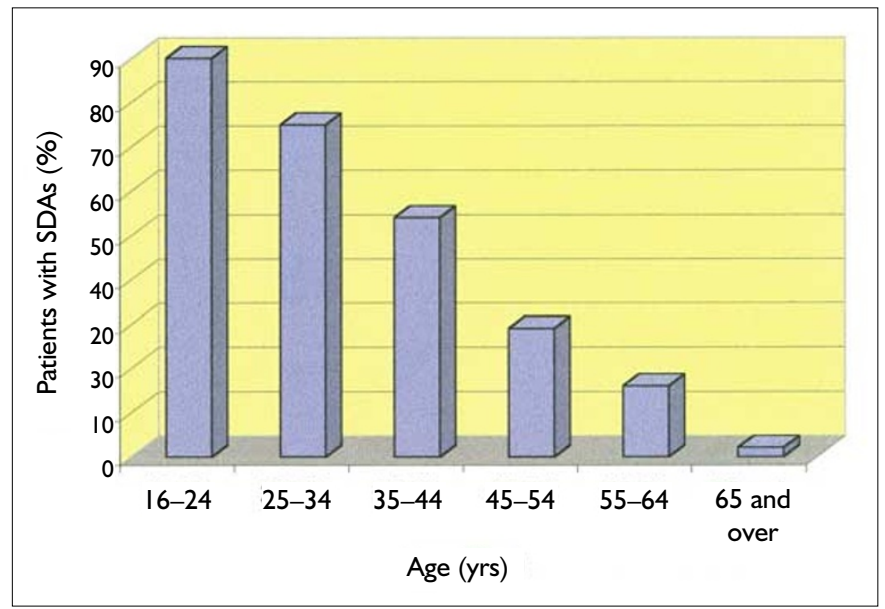

In the second article in the series there is an account of the real benefits that can accrue from wearing RPDs, as well as illustration of the tissue damage which can occur. This 'biological price' is particularly likely to be exacted if RPDs are not maintained adequately even if they are well-designed and constructed. It is therefore prudent for the dentist to consider
Fig. 4

For how many people is the SDA concept a viable proposition? The percentage of the UK adult population that possessed four 'good' quadrants was found to be $54 \%$. The relationship to age is shown in the chart. The number of people who might have a functional SDA falls dramatically with age. Nevertheless, there is a risk that a considerable number of older people could well be provided with RPDs on the advice of a dentist and yet fail to need them or even wear them.

the following questions before providing an RPD for any patient.

- 'Does the patient really want an RPD?' (the patient's assessment).

- 'Are the benefits likely to outweigh the possible damage that may occur?' (the dentist's assessment).

\section{RPDs and the elderly}

People are living longer and retaining more of their teeth into old age. This trend is likely to continue as the current middle-aged population, tomorrow's elderly, is showing a dramatic improvement in oral health compared with a similar group 20 years ago.

A nationwide survey showed that the majority of adults preferred to manage without a denture if several teeth were missing at the back of the mouth (Fig. 1). It also revealed that the older age groups were more negatively inclined towards the

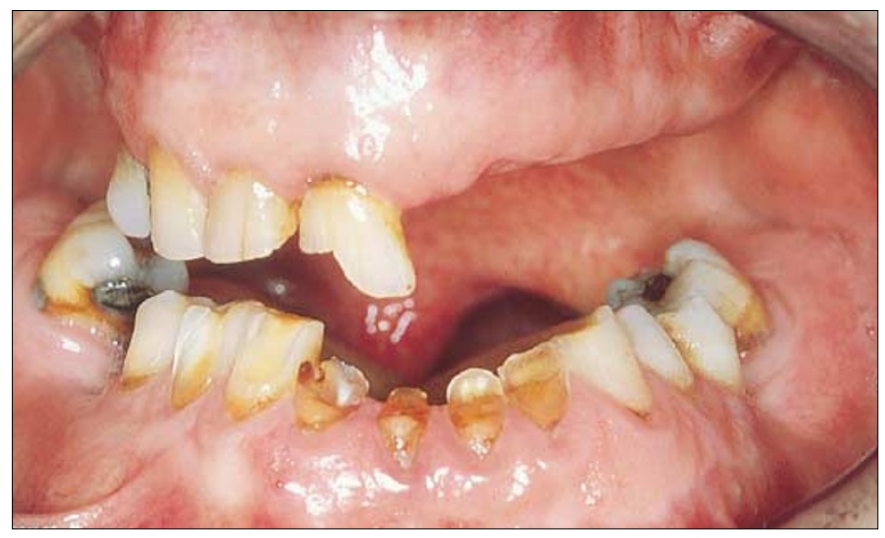

There is some evidence that in those patients who are susceptible to tooth wear, the problem may be more severe if the dentition is depleted and functional loading is concentrated on a small number of contacting teeth. If it is evident that tooth loss has created a deficiency in occlusal support, the provision of RPDs may help to stabilise the situation and prevent further deterioration.

Patients will often seek treatment because they are unhappy wearing of RPDs than younger individuals. It was as if the longer a person had managed to function adequately without an RPD, the greater the reluctance to resort to such a device. This potential barrier to the elderly accepting prosthetic treatment, together with the following specific problems, suggests that very careful thought is required before advising prosthetic treatment for the older population.

\section{Specific problems of the elderly in relation to the provision of RPDs}

Fig. 5 - Tooth wear

Tooth wear is an increasingly common finding, particularly in older patients. The initiating factor may be attrition, abrasion or erosion, and in more advanced wear a combination of these agents may be involved. It is important to establish a diagnosis if possible and institute measures to eliminate any causative factors, thus preventing further damage to the dentition.

with the appearance of worn teeth in the anterior region of the mouth. When the wear is moderate in extent, the teeth may be either maintained or restored with crowns and RPDs in order to stabilise the occlusal relationship and replace missing teeth. When wear is more severe, overlays may be incorporated in the denture design to provide a satisfactory aesthetic and functional result. 


\section{PRACTICE prosthetics}

\section{Fig. 6 - Tooth wear}

In extreme cases it may be more appropriate to consider the reduction of some teeth to serve as overdenture abutments. Root abutments can make a substantial contribution to the support of RPDs, particularly when the alternative would be an edentulous saddle area opposed by a substantial group of natural teeth.
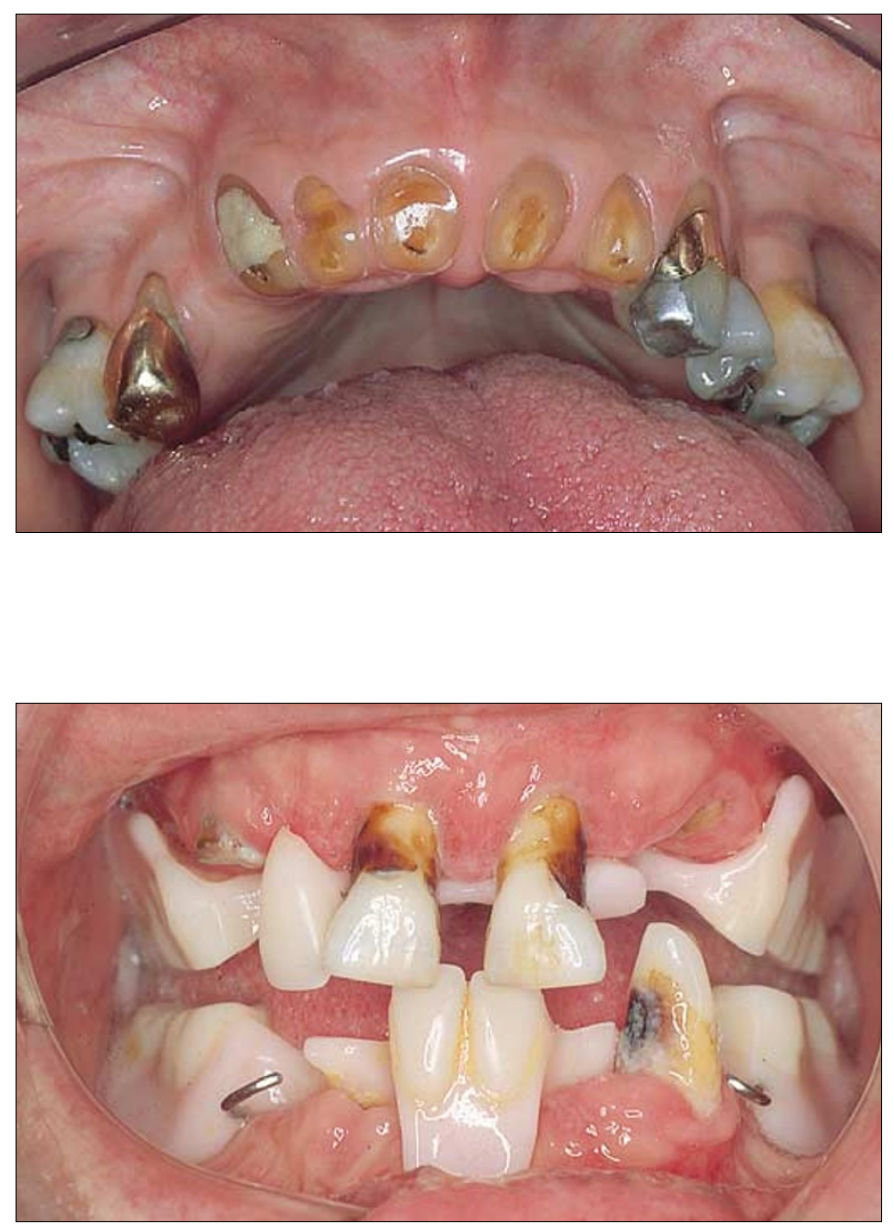

\section{Fig. 7 - Root surface caries}

Reference has previously been made to the tendency for RPDs to encourage the accumulation of plaque. This factor becomes particularly important in older patients as gingival recession may lead to the exposure of root structure and an increased risk of root surface caries. The situation will be aggravated further if the ability to maintain adequate plaque control becomes impaired for any reason and if the intake of fermentable carbohydrate increases as taste sensitivity declines and masticatory efficiency diminishes. It is therefore particularly important to ensure that dentures are designed to minimise contact with, or coverage of, vulnerable areas. For example, gingivally approaching clasps which cross and contact the exposed root surface should only be used if a careful evaluation indicates that the risk to a particular patient is insignificant.

\section{Patient assessment \\ General health status}

Age by itself is an imprecise guideline in the assessment of older patients. Of much greater importance is the effect that illness may have had on the ageing process. For this reason a detailed medical history is essential and should include a full list of any prescribed medications.

The state of health is an important factor to be considered when deciding whether or not to advise the provision of RPDs. Progressive infirmity or debility can have an adverse effect on the capacity to adapt and may explain the difficulty that some patients have in coming to terms with dentures, particularly if they have had no previous denture wearing experience. It is thus very important to determine what, if any, improvement in the state of health can be expected. The effect that health factors may have on the ability of patients to attend for treatment and subsequent maintenance must also be considered.

The wearing of even the best designed RPD is likely to be accompanied by an increase in plaque accumulation. If there is evidence of the patient having difficulty in undertaking maintenance procedures, the potential for damage may outweigh the likely benefits of the prosthesis in terms of function and aesthetics. For example, a patient may have difficulty in cleaning natural and artificial teeth because of failing eyesight. Alternatively, a drug-induced dry mouth with its associated reduction in the buffering capacity and volume of saliva can result in a dramatic increase in caries and periodontal disease.

\section{Desire and motivation}

As mentioned earlier, it is important to assess the degree to which patients are motivated to wearing RPDs. It is not uncommon to find that the request for prosthetic treatment comes from carers or members of the family, particularly if they feel that the patient's recovery from severe illness is being hampered by an inadequate dentition. Unless patients themselves wish to have RPDs and unless a high standard of plaque control can be achieved either by the patients themselves or by the carers, treatment is unlikely to be successful.

\section{Domestic circumstances}

Social factors should also be considered. For example, the absence of support from carers may pose difficulties for patients in attending for treatment or in complying with the necessary maintenance procedures.

\section{Assessment of existing RPDs}

The assessment of existing RPDs and an understanding of previous denture wearing experience should follow precisely the format described in Chapter 7 of our BDJ publication 'A Clinical Guide to Partial Dentures'. 


\section{PRACTICE prosthetics}

\section{Treatment options}

For the older patient, decisions on whether or not to provide RPDs and of balancing benefits of a prosthesis against potential

harm to the mouth may well be more critical than for a younger patient.
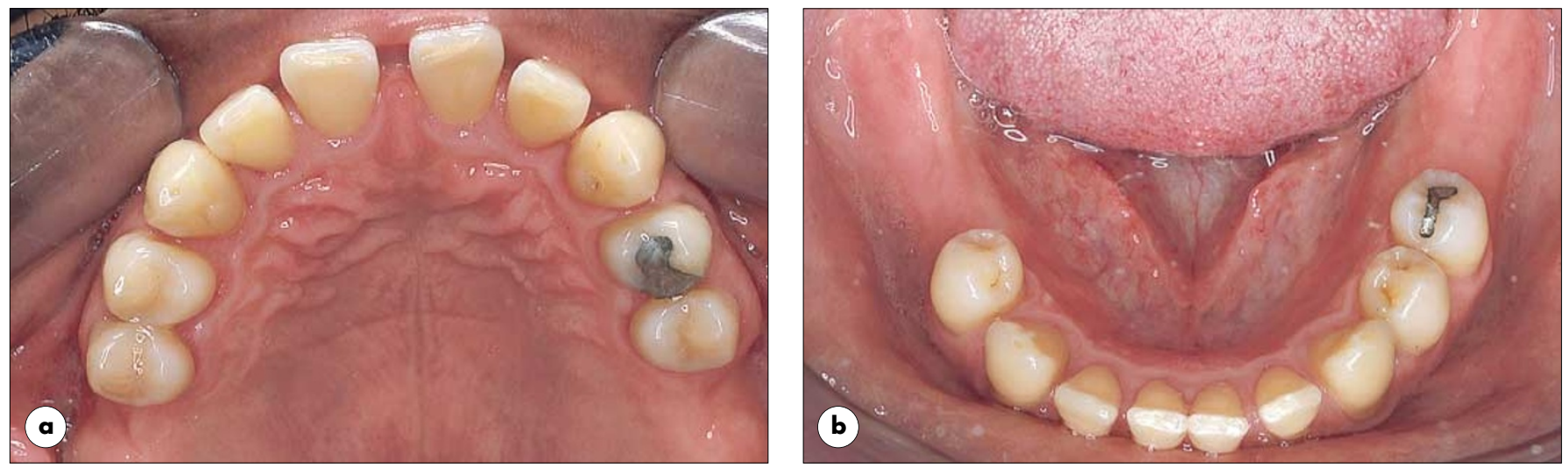

\section{Fig. 8a and $b-$ Treatment options}

If a shortened dental arch exists particular attention must be given to the possibility of simply maintaining the status quo rather than providing an RPD.

\section{Fig. 9 - Treatment options}

In this example the patient had no worries about appearance but had experienced difficulties in eating. By providing a mandibular RPD to improve masticatory ability, treatment that met the patient's specific concerns, it was possible to avoid an RPD in the maxillary arch.

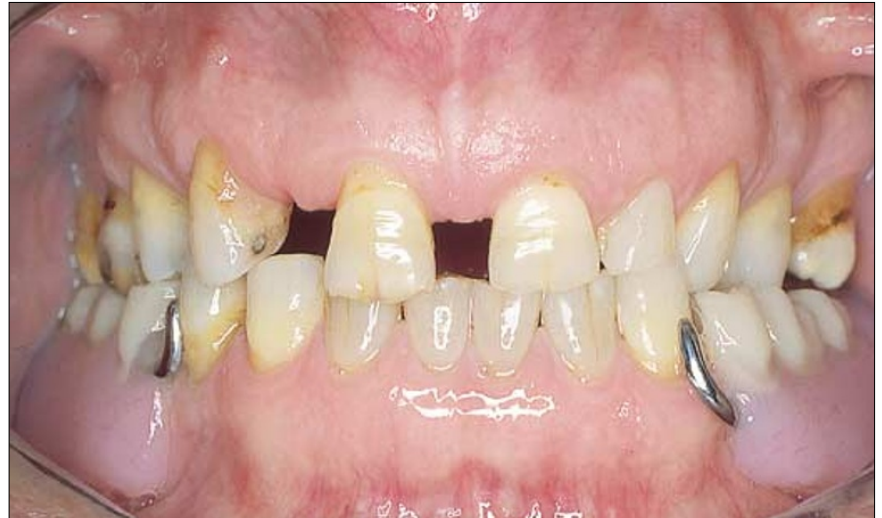

\section{Fig. 10 - Treatment options}

Where there has been extensive tooth loss, one of the most important decisions is whether or not to retain a selection of teeth to assist with the wearing of an RPD. Even if it appears that such an arrangement may have a limited life it is usually far preferable to extractions and the provision of complete dentures. In this example the remaining natural teeth helped to stabilise maxillary and mandibular RPDs. After a short time two of the maxillary teeth and both mandibular teeth were converted to overdenture abutments and continued to serve the patient faithfully for many years. Thus an effective 'pre-edentulous' state was preserved. Even if the eventual extraction of the remaining teeth is inevitable, their retention in the short term to stabilise an RPD can make a significant contribution to a successful transition of a patient to complete dentures.

\section{Further reading}

\section{Need and demand}

Basker R M, O'Mullane D M. Removable prosthodontic services related to need and demand. In Öwall B, Kayser A F, Carlsson G E. Prosthodontics: principles and management strategies. pp 223-235. London: Mosby-Wolfe, 1996.

Douglas C W, Gammon M D, Atwood D A. Need and effective demand for prosthodontic treatment. J Prosthet Dent 1988; 59: 94-104.

Fiske J, Davis D M, Frances C, Gelbier S. The emotional effects of tooth loss in edentulous people. Br Dent J 1998; 184: 90-93.

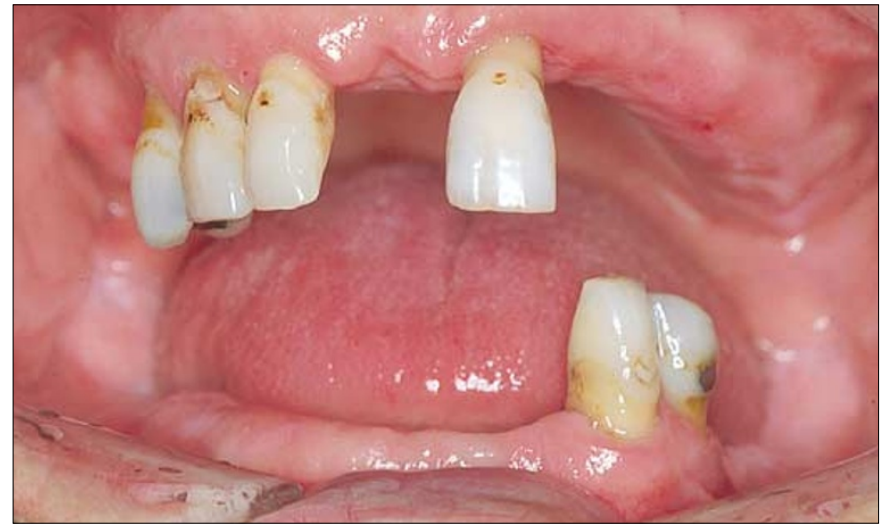

\section{RPDs and the elderly}

Drummond J R, Newton J P, Yemm R. Dental care of the elderly. London: MosbyWolfe, 1994.

Franks A S T, Hedegård B. Geriatric Dentistry. Oxford: Blackwell Scientific, 1973. Ralph J P, Basker R M. The partially edentulous patient. In Barnes I, Walls A (ed). Gerodontology. pp 127-134, London: Wright, 1994. 